Utah State University

DigitalCommons@USU

$1-1-2008$

\title{
Wide-Field Infrared Survey Explorer Cryogenic Support System Status and Thermal Test Results
}

Scott Schick

Brett Lloyd

Follow this and additional works at: https://digitalcommons.usu.edu/sdl_pubs

\section{Recommended Citation}

Schick, Scott and Lloyd, Brett, "Wide-Field Infrared Survey Explorer Cryogenic Support System Status and Thermal Test Results" (2008). Space Dynamics Lab Publications. Paper 83.

https://digitalcommons.usu.edu/sdl_pubs/83

This Article is brought to you for free and open access by the Space Dynamics Lab at DigitalCommons@USU. It has been accepted for inclusion in Space Dynamics Lab Publications by an authorized administrator of DigitalCommons@USU. For more information, please contact digitalcommons@usu.edu.

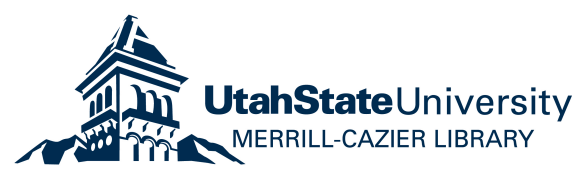




\title{
Wide-field Infrared Survey Explorer cryogenic support system status and thermal test results
}

\author{
Scott Schick ${ }^{1}$ and Brett Lloyd ${ }^{2}$ \\ ${ }^{1}$ Practical Technology Solutions, Inc. North Logan, Utah 84341, USA \\ ${ }^{2}$ Space Dynamics Laboratory North Logan, Utah 84341, USA
}

\begin{abstract}
The Wide-Field Infrared Survey Explorer (WISE) is a MIDEX mission that is being developed by the Jet Propulsion Laboratory (JPL) to address several of NASA's Astronomical Search of Origins (ASO) objectives. Space Dynamics Laboratory/ Utah State University is providing the cryogenically cooled infrared instrument. Cooling for the instrument optics and focal planes is provided by a dual-stage solid hydrogen cryostat. Driving requirements for the cryogenic subsystem are: a seven-month lifetime and operating temperatures of less than $17 \mathrm{~K}$ for the optics, $32 \mathrm{~K}$ for the $\mathrm{HgCdTe}$ focal planes, and $7.8 \mathrm{~K}$ for the Si:As focal planes. This paper provides an overview of the dual-stage hydrogen cryogenic subsystem status and discusses the results of the thermal performance testing.
\end{abstract}

Keywords: Cryogenic cooling, solid hydrogen, cryostat, thermal, WISE

\section{INTRODUCTION}

The Wide-field Infrared Survey Explorer (WISE) mission is a NASA medium explorer project (MIDEX) being developed with the Jet Propulsion Laboratory (JPL). The primary goal of WISE is to provide an all-sky survey in four infrared imaging channels covering from 2.8 to $25 \mu \mathrm{m}$ with over 500,000 times the sensitivity of previous all-sky surveys. In particular, WISE will: find the most luminous galaxies in the Universe, find the closest stars to the Sun, detect Main Belt asteroids larger than 3-km, contribute to our understanding of star and galaxy formation, provide an essential catalog for the James Webb Space Telescope, and provide a lasting research legacy. WISE will be launched into a 530-km, sun-synchronous, polar orbit in November 2009. During the seven-month mission, WISE will image each section of sky a minimum of eight times. These images will be combined to provide a single, high sensitivity full-sky survey. This paper will present a brief overview of the cryogenic support system (CSS) followed by a discussion of the thermal testing. More detailed descriptions of the WISE instrument and the CSS design have been published previously ${ }^{[1]}$.

\section{INSTRUMENT OVERVIEW}

The WISE instrument (Fig. 1) is a cryogenically cooled infrared instrument consisting of a 40-cm aperture telescope, scan mirror, and beam splitters to separate light into four bands. Light is measured using four focal planes, two 32-K mid-wave infrared (MWIR) HgCdTe focal plane arrays (FPA) and two 7.8-K long-wave infrared (LWIR) Si:As FPAs. The entire optical assembly is housed within the two-stage solid-hydrogen cryostat. The sole purpose of the primary tank is to cool the two LWIR FPAs, while the larger secondary tank cools the rest of the optics to less than $17 \mathrm{~K}$. The optical assembly mounts to a single interface flange within the cryostat for simple and reliable integration and ease of removal during the testing sequence. The primary tank is connected to the LWIR FPMAs via a flexible, shrink-fit style, thermal link. The link consists of beryllium end fittings attached to copper braids. A deployable aperture cover hermetically seals the vacuum shell during ground operations and is jettisoned on-orbit to open the telescope aperture to view space. The WISE instrument is thermally isolated from the spacecraft, allowing the outer shell to be passively cooled to below $200 \mathrm{~K}$ on orbit to limit parasitic heat loads into the solid hydrogen.

\section{CRYOGENIC SUPPORT SYSTEM CONFIGURATION}

The essential features of the WISE cryogenic support system are highlighted in Fig. 1 and have been discussed in detail in a previous paper ${ }^{[2]}$. A brief description is given here to provide context for the test discussion.

Astronomical and Space Optical Systems, edited by Penny G. Warren, Cheryl J. Marshall,

Robert K. Tyson, Michael Lloyd-Hart, James B. Heaney, E. Todd Kvamme, Proc. of SPIE

Vol. $7439,743917 \cdot$ C 2009 SPIE · CCC code: 0277-786X/09/\$18 · doi: 10.1117/12.825743

Proc. of SPIE Vol. $7439743917-1$ 


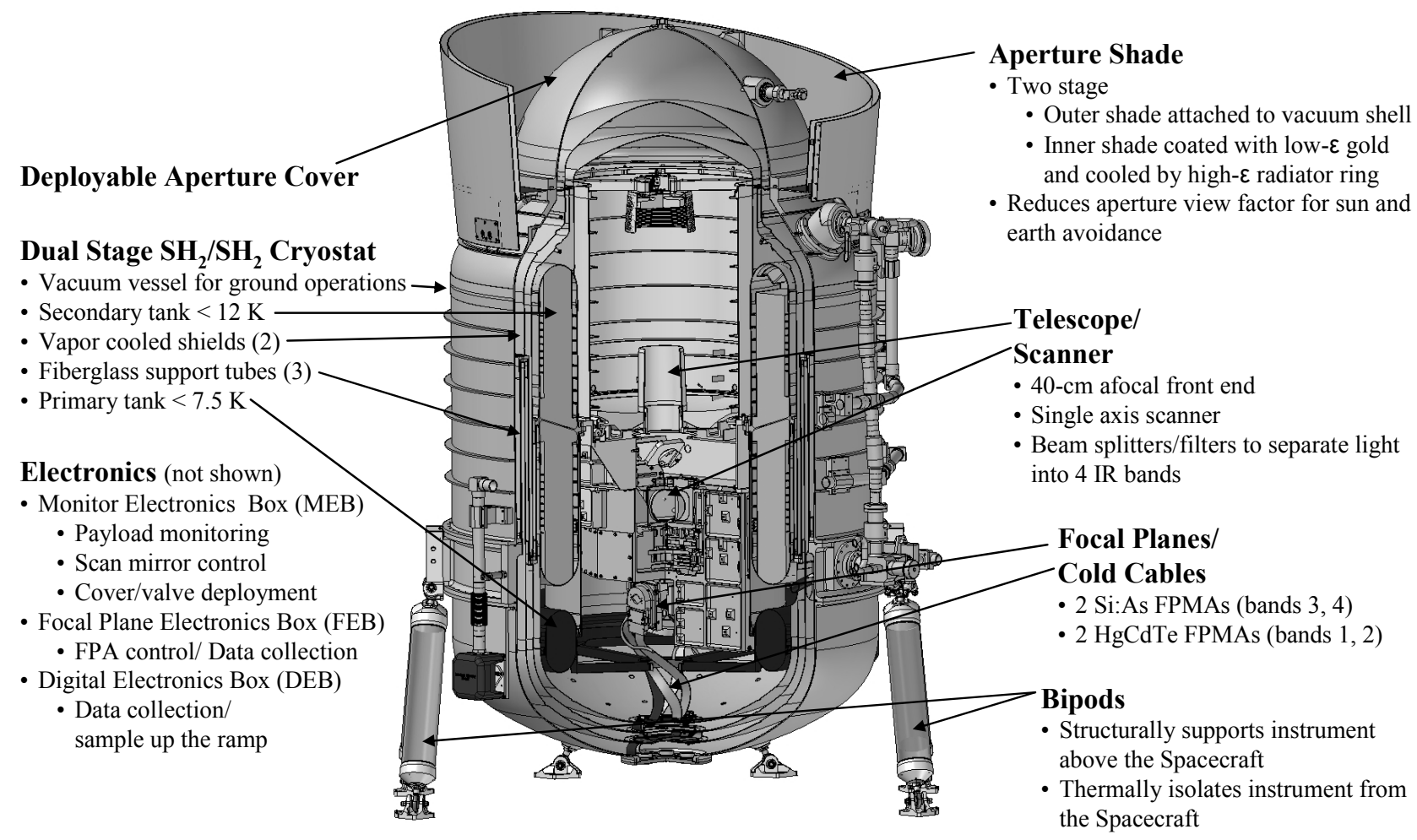

Fig. 1. WISE instrument configuration

The cryostat, built by Lockheed Martin's Advanced Technology Center (LM-ATC), includes two cryogen tanks that contain solid hydrogen for cooling on-orbit. The 186-liter secondary tank is used to cool the majority of the optics to below $17 \mathrm{~K}$ and to intercept the bulk of the parasitic heat loads, thus reducing the heat loads on the primary tank to insignificant levels. The 24.4-liter primary tank is used solely to cool the Si:As focal planes modules to $7.8 \pm 0.5 \mathrm{~K}$. Both tanks are filled with $1.7 \%$ dense, 1100 aluminum foam to provide heat exchange from the hydrogen to the tank walls. The design allows for both ground and on-orbit operations. The cryostat requirement is to provide cooling to support a seven-month mission; the cryostat was designed to extend this mission and to provide extra life as risk mitigation during the cryostat fabrication and test in case the performance was not as expected. As will be shown later, the expected life is short of the early predictions due to imperfections in the insulation and non-ideal connections of the shields to the vapor cooling.

\subsection{Thermal Isolation, Vapor Cooled Shields \& Vacuum Enclosure}

Thermal isolation between temperature zones is provided by fiberglass tubes, silk net/double aluminized mylar multilayer insulation (MLI), two vapor-cooled shields (VCS) (including one that is also radiation-cooled), and low conductance lead wires for the instrumentation.

The tanks and shields are supported using three nested fiberglass tubes. The tubes are an S2-E glass cloth weave with the fill direction in the axial direction to limit the conduction heat loads. A fourth fiberglass tube mechanically connects and thermally isolates the primary tank from the secondary tank.

Two VCSs are placed between the outer shell and tanks to reduce the parasitic heat loads to the cryostat. Each shield is attached to the hydrogen vent lines using a small copper braid. During testing, it was found that the temperature drop across this small link did not provide sufficient connection to the vent line thus limiting the effectiveness of the cooling at each shield. A radiator plate, incorporated on the outer VCS in the aperture plane, provides additional cooling at this temperature zone.

The cryostat is hermetically sealed on the ground by a ring-stiffened vacuum shell and a deployable aperture cover. The cryostat attaches to and is isolated from the spacecraft by four pairs of bipods. The bipod struts are S2 fiberglass tubes 
with spherical ball end fittings that provide the requisite support through launch, alignment and stability during operation, and thermal isolation from the ambient spacecraft structure.

\section{CRYOGENIC SUPPORT SYSTEM STATUS}

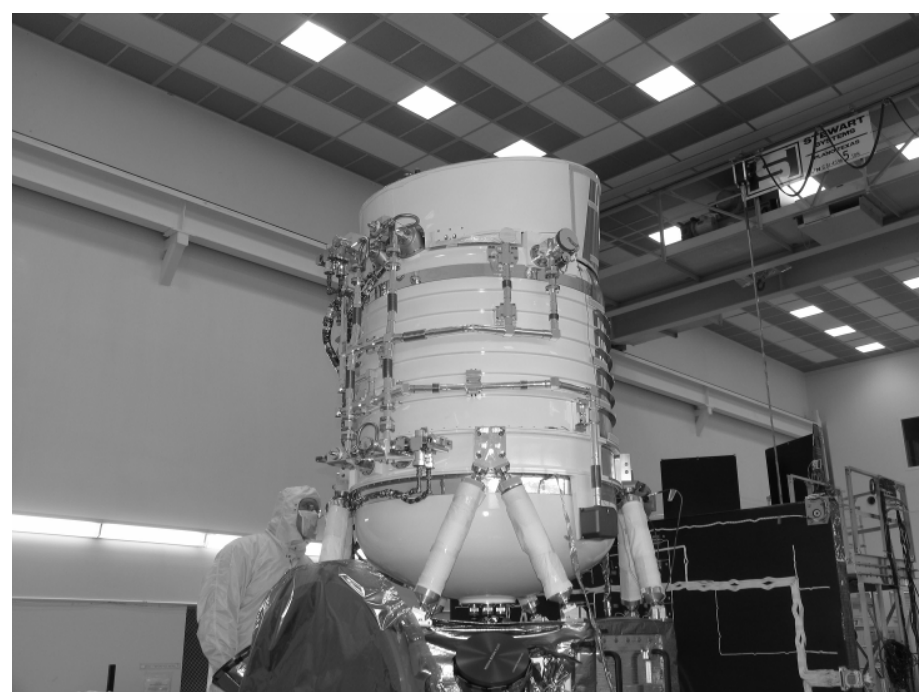

Fig. 2. WISE instrument mated to the spacecraft
The flight optics have been integrated with the cryostat, and the flight instrument has completed testing. Most recently, the instrument was integrated with the spacecraft and is undergoing system-level functional and environmental tests (Fig. 2).

After the cryostat was delivered to SDL in February 2008, protoflight level structural testing was performed with a mass simulator telescope installed. Testing was done cold but without hydrogen in the tanks. Since the hydrogen is very light, the lack of hydrogen did not impact the test significantly. The loads were increased slightly to put the same stresses on the support tubes.

Instrument functional testing was performed using liquid helium in an open loop system around the tanks to achieve the flight temperatures without the risks associated with solid hydrogen.

Acceptance vibration testing was performed after the flight optics were installed inside the cryostat. Following the vibration test, an acceptance level thermal test using hydrogen was performed on the flight instrument. The primary purpose of this paper is to describe the thermal performance testing of the flight instrument ground hold and heat rate tests. During this test, procedures were worked through and red-lined as the cryostat was cooled and solid hydrogen was loaded into the cryostat.

Following the successful acceptance level vibration test, the cryostat was moved to the Wyle hydrogen test facility at San Bernardino, CA. After arriving, the GSE was set up and checked out at the test site in preparation for the hydrogen fill and heat rate testing. Fig. 3 shows the test setup. Note that the tests were performed at an outdoor site at ambient conditions. The aperture cover was in place to seal the vacuum space in order to facilitate a safe fill and heat rate test.

\subsection{Hydrogen Fill Operations}

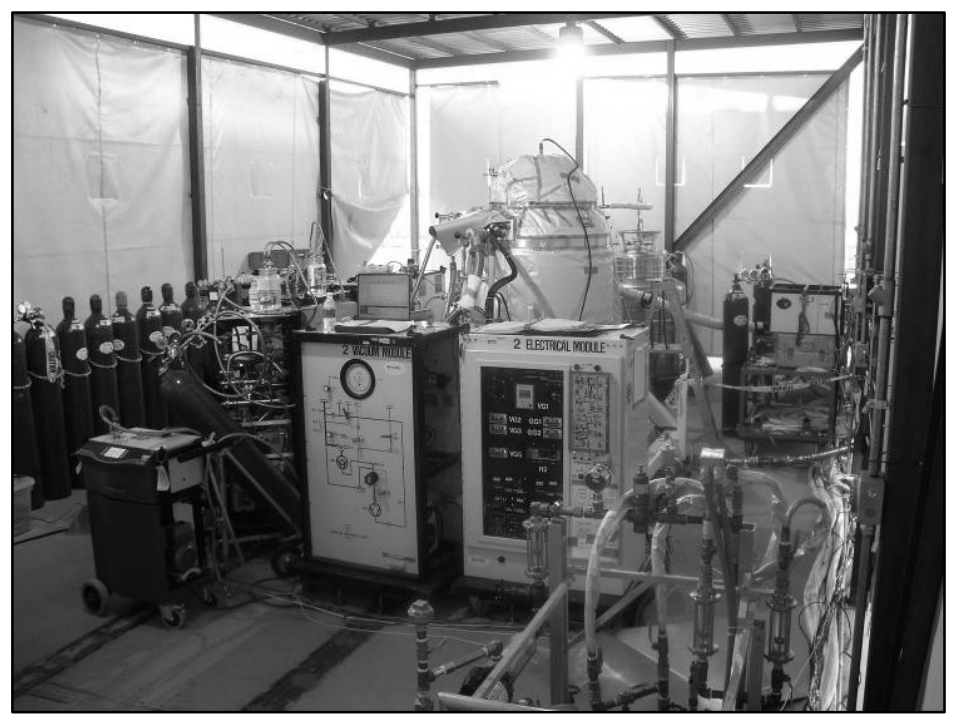

Fig. 3. Hydrogen test setup
Procedures for cryostat operation were developed based on previous solid hydrogen instrument projects. The cryostat was filled using a gas-fill technique that was selected to keep the flight plumbing mass low. Liquid helium was cycled through the cooling coils around the tanks while gaseous hydrogen flowed into the tank. Once inside the tank, the hydrogen gas condensed into a liquid to slowly fill the tank volume. The fill quantity was measured using two mass flow meters (one primary, one backup). After the tanks were filled, the hydrogen supply was shut off; the helium continued to flow to freeze the hydrogen.

The design of the cryostat allows for enough ullage space in the tanks to allow complete melting of the hydrogen for safe ground operations. The secondary tank was filled with $13.94 \mathrm{~kg}$ of hydrogen and the hydrogen was frozen and cooled 
to approximately $6 \mathrm{~K}$. The primary tank was filled with $1.8 \mathrm{~kg}$ of hydrogen in a similar fashion. Both tanks had less usable volume than intended in the design. The secondary tank was filled with the correct quantity of hydrogen, but the primary tank was filled with $0.1 \mathrm{~kg}$ less hydrogen than designed.

\subsection{Ground Hold Test}

Following the fill, a ground hold test was performed to measure the warm-up rate of the hydrogen. The ground hold capability is very important. While on the ground, the hydrogen does not vent from the tanks. Thus, if the tanks are not being actively cooled, the solid hydrogen slowly warms or melts. The non-cooling periods are necessary to allow ground operations to occur during integration with the launch vehicle. Cooling continued on the cryostat for more than 48 hours after the fill to subcool the cryostat before disconnecting for the ground hold test. After this sub-cooling period, the cryostat ground hold measurement was made. The time to triple point from the time the liquid helium coolant was stopped was measured as 51.8 hours. The hydrogen was allowed to melt for an additional 48 hours (100 hrs total) to verify the time needed for ground operations. These values are sufficient and have ample margin to safely process the WISE cryostat at the launch site and launch the cryostat to orbit aboard the Delta II. The 52-hour ground hold exceeds the 44-hour requirement that was placed on the cryostat by the payload team to allow for two, successive day, launch attempts before needing to re-cool (See Fig. 4).

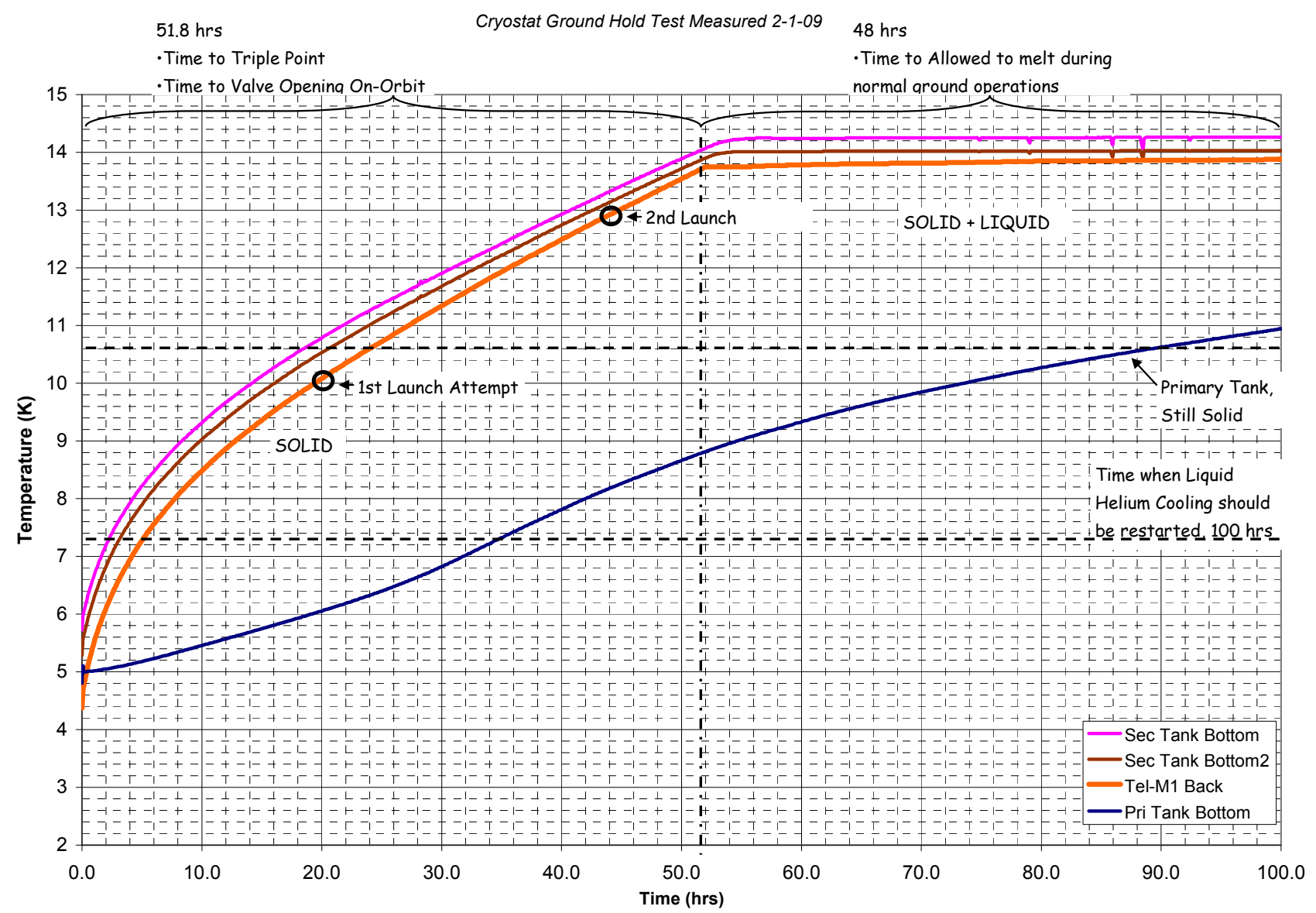

Fig. 4. WISE ground hold time frame indicating the state of the hydrogen

\subsection{Heat Rate Test}

After the ground hold measurement was made, the hydrogen was again frozen with liquid helium. The GSE was reconfigured to pump on each tank simultaneously to simulate the vacuum of space. This allows the hydrogen to sublime and the vapor to exit through the plumbing and provide vapor cooling to each shield in a flight-like manner. The heat rate test was performed on the cryostat with the door on and with the vacuum shell at ambient temperatures. The primary tank was pumped on directly while the secondary tank was pumped through a ground set of the on-orbit nozzles to ensure the secondary cryogen tank would stay under $13 \mathrm{~K}$ as required. In the test condition, the aperture door is one of the 
driving heat loads on the cryostat. During the test, the instrument was turned on and the shields and tubes allowed to equilibrate for several days until a steady condition was reached and the heat rate could be measured. The result of the measurement was a ground heat rate of $1.7 \mathrm{~W}$ with an average $285 \mathrm{~K}$ vacuum shell.

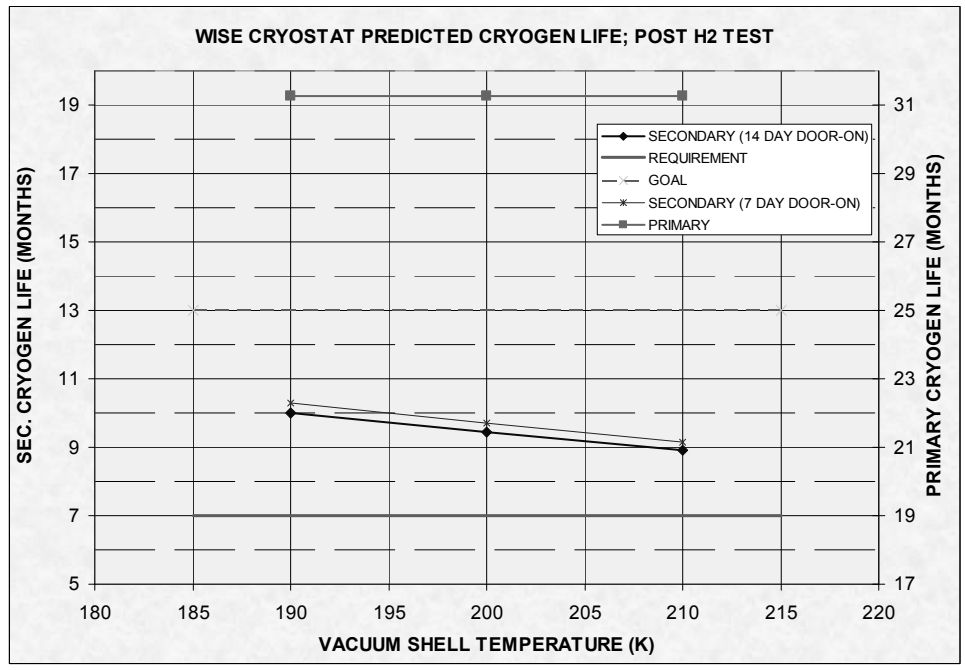

Fig. 5. On-orbit cryogen life predictions

During the heat rate test, all required instrument temperatures were met even though the shell was at ambient temperature. The optics stayed under $17 \mathrm{~K}$ with a $12.8 \mathrm{~K}$ hydrogen sink that is expected to be approximately $2.5 \mathrm{~K}$ colder onorbit. The Si:As focal planes operated at 7.1 to $7.2 \mathrm{~K}$ during the test. They are expected to operate at approximately $7.3 \mathrm{~K}$ on orbit, after accounting for an annealing temperature offset.

\subsection{Lifetime Predictions}

Model predictions prior to the test were $54 \mathrm{hrs}$ for the ground hold and $1.3 \mathrm{~W}$ for the heat rate. The thermal model was then correlated to the ground test results to better predict on-orbit lifetime with the expected shell temperature and door removed. Lifetime predictions, shown in Fig. 5, were made using the test data, fill quantities, and modeling the expected $191 \mathrm{~K}$ onorbit shell temperature from the latest flight system modeling information. The results indicate that the secondary tank hydrogen should last 10 months (304 days). The predicted duration of the primary tank hydrogen significantly exceeds the secondary at 31 months. The long primary life was obtained because the Si:As focal plane heat dissipation was much less than expected. These lifetime predictions exceed the seven-month requirement but fall short of the 13-month goal set by the project. The predicted on-orbit heat map is shown in Fig. 6.

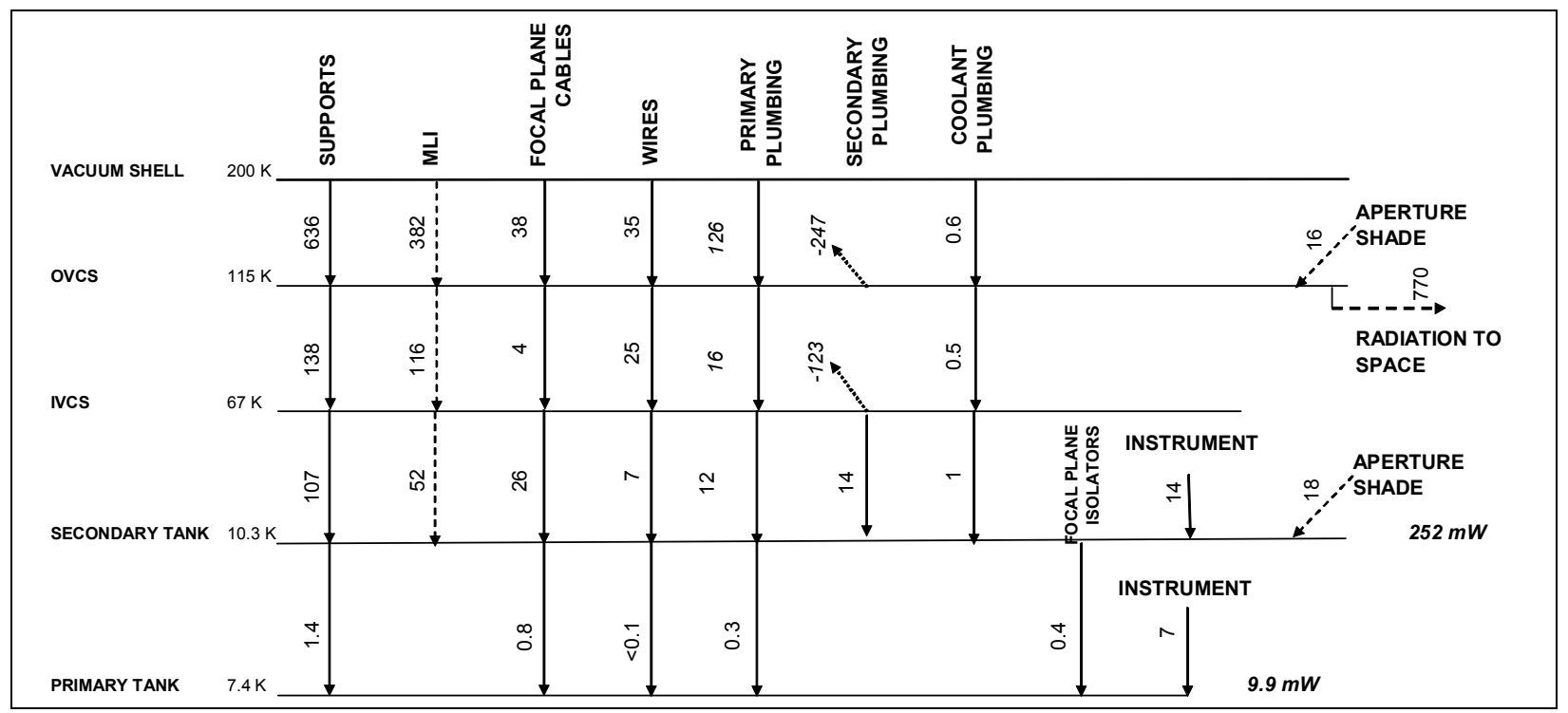

Fig. 6. Heat map of the on-orbit case.

\subsection{Annealing Tests}

During the mission, the Si:As focal planes require annealing by heating the arrays above $15 \mathrm{~K}$ to remove latent images on the array. Since this impacts the hydrogen temperature, annealing tests were performed near the end of the heat rate testing. The tests showed that with the annealing heaters on for 90 seconds, the FPAs warmed above $15 \mathrm{~K}(15.8 \mathrm{~K}$, minimum), requiring approximately 23 minutes to re-stabilize to operational temperatures (See Fig. 7). This time to 
stabilization varies with the hydrogen level in the tank. The maximum predicted stabilization time is approximately 100 minutes at $25 \%$ cryogen (See Fig. 8 ).

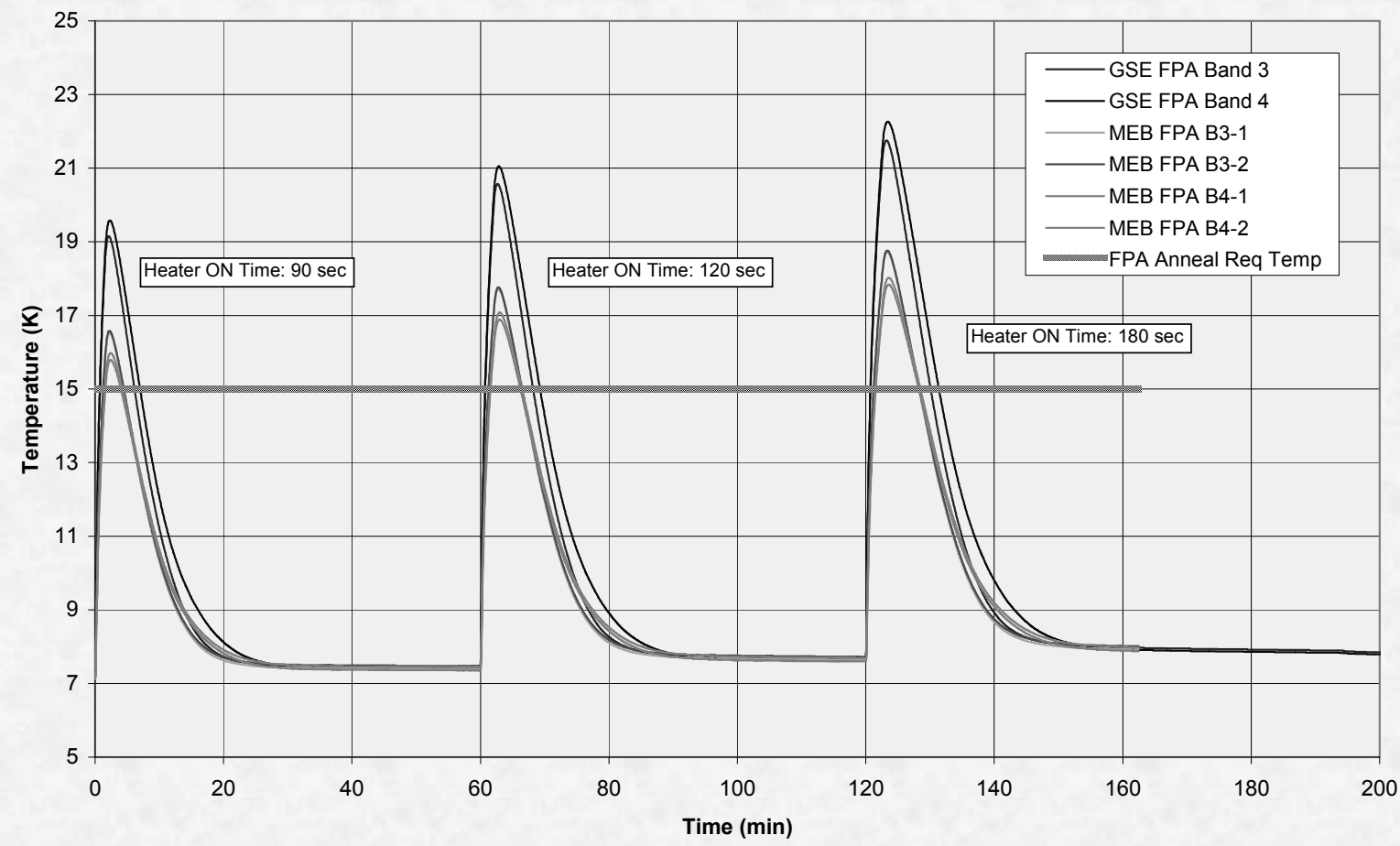

Fig. 7. Measured Si:As FPA and primary tank temperatures during annealing tests. Temperature data from both the cryogenic GSE temperature monitoring system and Engineering Unit MEB (Monitor Electronics Box) are shown.

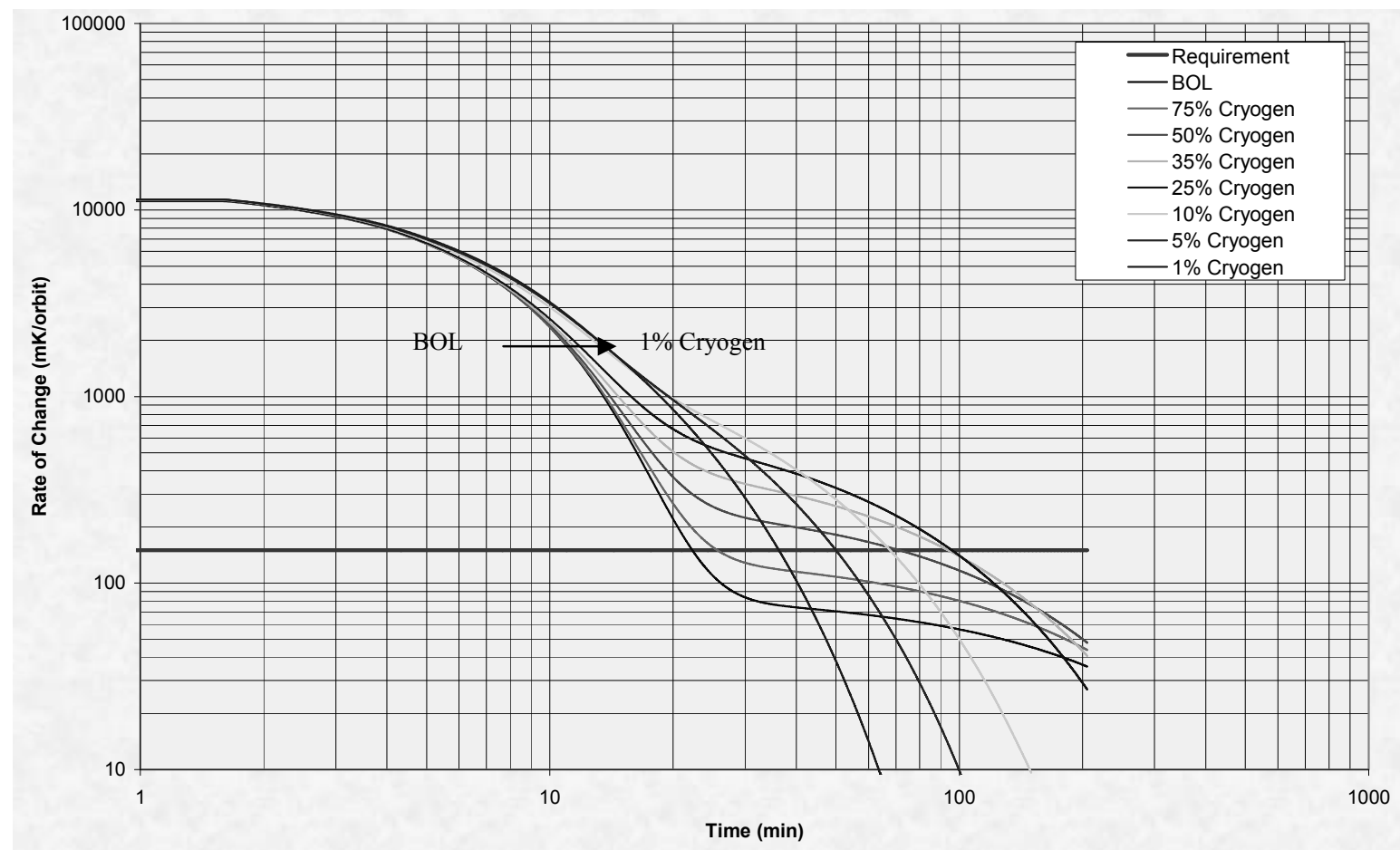

Fig. 8. Estimated stabilization time of the Si:As FPA temperature due to an annealing cycle. 


\subsection{DISCUSSION OF HEAT RATES}

The degradation in on-orbit lifetime from the predicted values was a combination of several factors in the as-built instrument. The focal plane cable conductance, the MLI blanket and support conductance were all slightly higher due to the vapor cooled shields running warmer than expected. The shield temperatures were warmer because they were not anchored to the orbit vent heat exchangers as well as expected. This reduced the effectiveness of the vapor cooling. There are also some non-ideal radiation couplings at the front end MLI blanket edges that increased the blanket temperatures and thus reduced the blanket performance. Finally, the aperture door, which has been a known thermal problem since the first cold test at SDL, increases the heat load significantly during the first two weeks. All of these contributors lead to the approximately $20 \%$ lower expected on-orbit lifetime.

\section{CONCLUSION}

All thermal requirements are being met with the cryostat with comfortable margin. The hydrogen test provided a tremendous amount of information about the cryostat in the flight configuration. Hold times from the start of disconnect to triple point and then through approximately $25 \%$ melt were 52 hours and 100 hours, respectively. The measured value exceeded the 44-hour requirement by eight hours, providing some margin in getting the hydrogen tank valves open after the second launch attempt. During ground operations, it is allowable to have the hydrogen go into triple point and melt for up to 48 hours. This extends the ground operations working time from the beginning of the LHe disconnect to 100 hours, at which time the liquid helium cooling must be restarted. All of the measured durations allow sufficient time to prepare and launch the flight system.

Ground test information was used to update the thermal model and provide new estimates for the cryogen on-orbit lifetime. The updated prediction for the on-orbit life with the expected $191 \mathrm{~K}$ shell temperature is 10 months for the secondary tank and 31 months for the primary tank. The limiting factor is the secondary tank which has a $43 \%$ margin over the 7-month requirement. This will allow time for taking additional data on-orbit.

\section{ACKNOWLEDGEMENTS}

The authors wish to acknowledge and thank Lockheed Martin Advanced Technology Center in Palo Alto, CA for their support and hard work building the cryostat for WISE. The authors wish to acknowledge the significant inputs from Mr. Greg Bell, who led the development of the test procedure and provided assistance and significant input on the test operations. They also wish to thank NASA and JPL for their support of the WISE program. The authors also appreciate the significant help from Mr. Joel Drake in putting this paper together and editing.

\section{REFERENCES}

[1] Larsen, M., Latvakoski, H., Mainzer, A., Schick, S., and Drake, J., "Wide-field infrared survey explorer science payload update," in Proc. SPIE Space Telescopes and Instrumentation, Vol. 7010 (2008)

[2] Naes, L., Lloyd, B., and Schick, S., "WISE cryostat design overview and build status," in Advances in Cryogenic Engineering, Transactions of the Cryogenic Engineering Conference, vol. 53, (2008) 